\title{
Design of Moving Target Detection and Tracking System Based on the Improved Optical Flow Method
}

\author{
Jun Gui \\ Information Science and Engineering School \\ Shenyang University of Technology \\ Shenyang, China \\ 784828033@qq.com
}

\author{
Tiansi Ma, Lijun Liu \\ Information Science and Engineering School \\ Shenyang University of Technology \\ Shenyang, China \\ jungui@hotmail.com
}

\begin{abstract}
Moving target detection is an important content of the visual field of research,This paper designs a system based on the Linux embedded platform to realize the moving target detection and tracking. This system choose the optical flow method as the algorithm, which is one of the important algorithms in the computer vision field, it can be achieved on moving targets detection and tracking. The experimental result shows that this system can be achieved on moving target detection and tracking.
\end{abstract}

Keywords-optical flow; moving target detection; Inux;

\section{INTRODUCTION}

Present systems are generally based PC or DS P as the hardware platforms, which can achieve superior performance and receive good results, but the price is very high. We choose ARM chips S3C2410X as the platform, it can meet all the functions on the des ign and pay the low price, costeffective. Because of Linux has many advantages such as open source, good network function, high safety, strong portability, we choose the linux 2.6 kernel system.

The algorithms of moving target detection and tracking are improved quickly. Temporal diference method, background subtracion method and optical flow method are always used in the moving target detection and tracking now.

\section{OPTICAL FLOW THEORY}

In recent yeas, the sch olars at home and abroad ar e making efforts to the development of the optical flow technology,they present a lot of new optical flow calculation method and improve the existing optical flow algorithm at the same time. In the computer vision field,there is some room for improvement and great prospects for development to the optical flow. Optical flow algorithm differs from the temporal diference method ,background subtracion method, it has the advantage that it is able to detect the outline of the complete of the moving target. When the moving target moves behind something, for example, the trees, the algorithm can not make the camera follow the moving target,and the alg orithm needs a lot of calculation, so there are a lot of problems in real-time. All of these problem can be solved with several methods.

The basic principle of opti cal flow method[3][4][5] is that it give a velocity vector to each pixel in the image,all of these velocity vectors are composed of the vector field.Every point on the $3 \mathrm{D}$ object and on the image is one to one.The image are ab le to be analyzed dynmamically at so me moment.When it don'te xist moving target in the background,the vector field of the image is continuous,on the contrary, if there are moving objects in the background, the vector field of the image is not continuous, it is able to detect the position of the object.

In this system, it ch oose the Lucas-Kanade algorithm[1][2] which is one of most popular and most commonly used optical flow method. Lucas-Kanade algorithm is first presented by Bruce D Lucas and Takeo Kande(30),it assume that the light does not chang ground the pixel,the speed of the vector on the image in the $\mathrm{X}, \mathrm{Y}$ direction is defined as $\left(V_{x}, V_{y}\right)$, they must satisfy the following equation:

$$
\begin{aligned}
& I_{x}\left(q_{1}\right) V_{x}+I_{y}\left(q_{1}\right) V_{y}=-I_{i}\left(q_{1}\right) \\
& I_{x}\left(q_{2}\right) V_{x}+I_{y}\left(q_{2}\right) V_{y}=-I_{t}\left(q_{2}\right) \\
& \vdots \\
& I_{x}\left(q_{n}\right) V_{x}+I_{y}\left(q_{n}\right) V_{y}=-I_{t}\left(q_{n}\right)
\end{aligned}
$$

The $\mathrm{q}_{1}, \mathrm{q}_{2}, \ldots \mathrm{q}_{\mathrm{n}}$ are the pixel point in the image,the $\mathrm{I}_{\mathrm{x}}\left(\mathrm{q}_{1}\right), \mathrm{I}_{\mathrm{x}}\left(\mathrm{q}_{1}\right), \mathrm{I}_{\mathrm{t}}\left(\mathrm{q}_{1}\right)$ are the partial derivative of the pixel point on the image in the $\mathrm{x}, \mathrm{y}$ direction.Presenting the equations set in maxtrix form like this, $\mathrm{Av}=\mathrm{b}$, that is:

$$
A=\left[\begin{array}{ll}
I_{x}\left(q_{1}\right) & I_{x}\left(q_{1}\right) \\
I_{x}\left(q_{2}\right) & I_{x}\left(q_{2}\right) \\
\vdots & \vdots \\
I_{x}\left(q_{n}\right) & I_{x}\left(q_{n}\right)
\end{array}\right], v=\left[\begin{array}{l}
v_{x} \\
v_{y}
\end{array}\right], b=\left[\begin{array}{l}
-I_{t}\left(q_{1}\right) \\
-I_{t}\left(q_{2}\right) \\
\vdots \\
-I_{t}\left(q_{n}\right)
\end{array}\right]
$$

Solving equations by least squares cubic method is that:

$$
\left[\begin{array}{l}
V_{x} \\
V_{y}
\end{array}\right]=\left[\begin{array}{cc}
\sum_{i} I_{x}\left(q_{i}\right)^{2} & \sum_{i} I_{x}\left(q_{j}\right) I_{y}\left(q_{i}\right) \\
\sum_{i} I_{x}\left(q_{i}\right) I_{y}\left(q_{i}\right) & \sum_{i} I_{y}\left(q_{i}\right)^{2}
\end{array}\right]^{-1}\left[\begin{array}{l}
-\sum_{i} I_{x}\left(q_{i}\right) I_{i}\left(q_{i}\right) \\
-\sum_{i} I_{y}\left(q_{i}\right) I_{i}\left(q_{i}\right)
\end{array}\right]
$$

\section{HARDWARE PLATFORM}

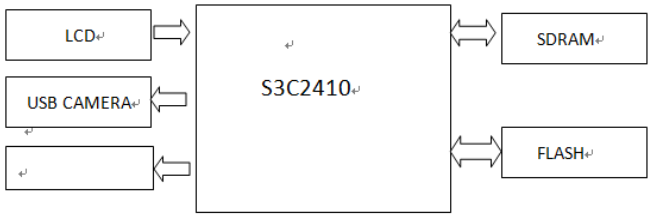

Figure 1.System Hardware Platform 
The Hardware structure is sho wn in F igure 1. The profecessor uses the $\mathrm{S} 3 \mathrm{C} 2410$, the proc essor integrates an ARM company ARM920T core,32-bit microcontrollers, its performance is excellent.It receives the images from the USB camera,and then transfer the $i$ mages to $t$ he Linux system area,in this area the images are analyzed and sended to the LCD display at the same time.

\section{PROGRAM FLOW CHART}

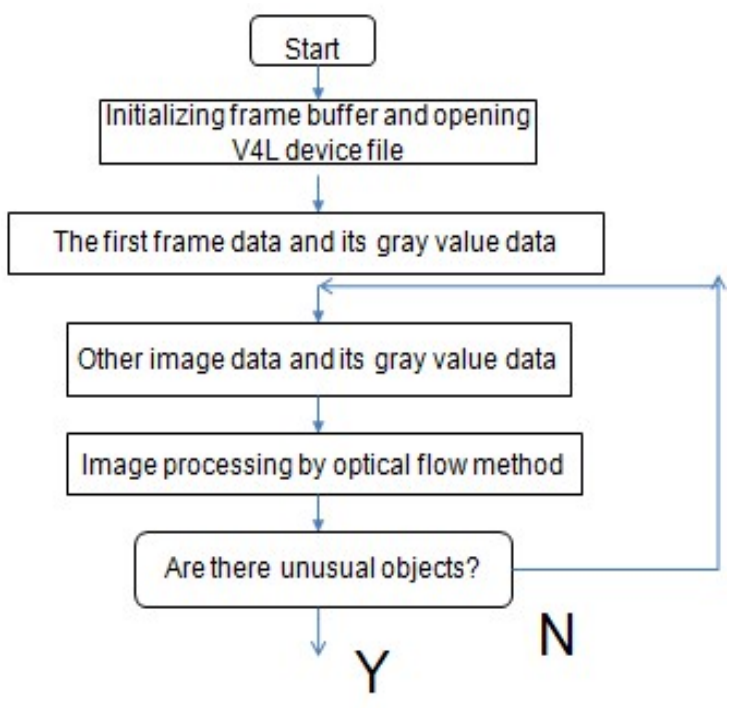

Figure 2. Program flow chart

\section{EXPERIMENTAL RESULTS AND ANALYSIS}

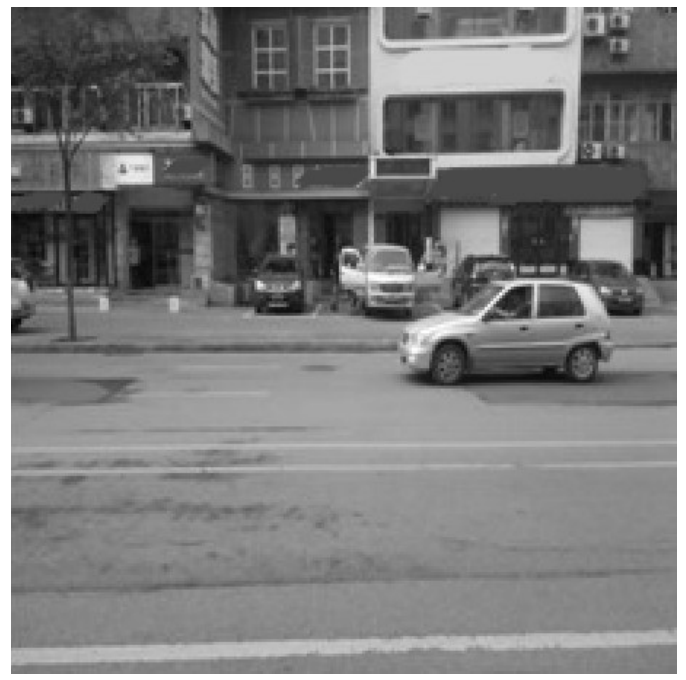

Figure 3. the First frame image

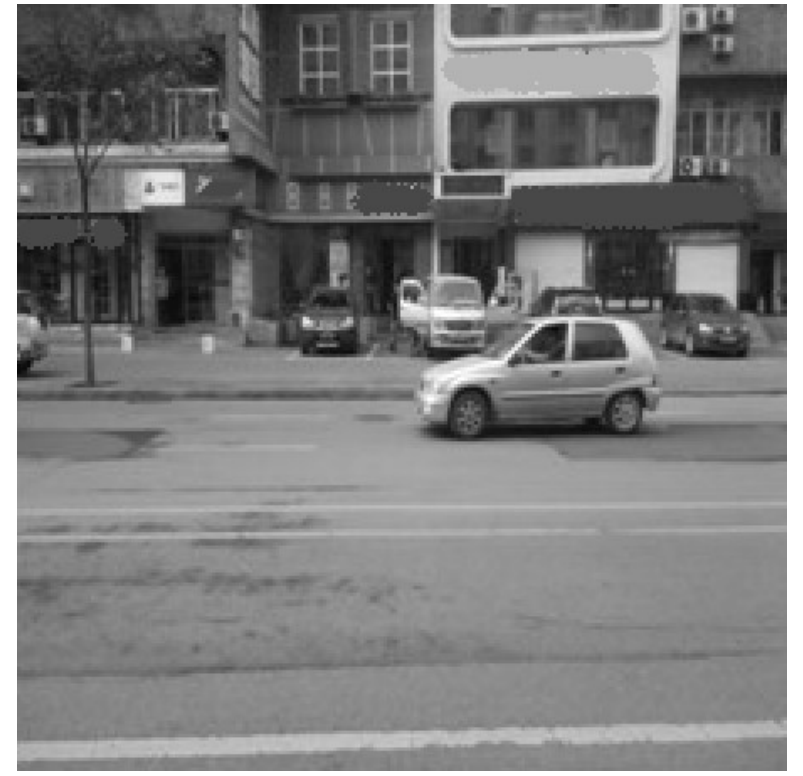

Figure 4.The Second frame image

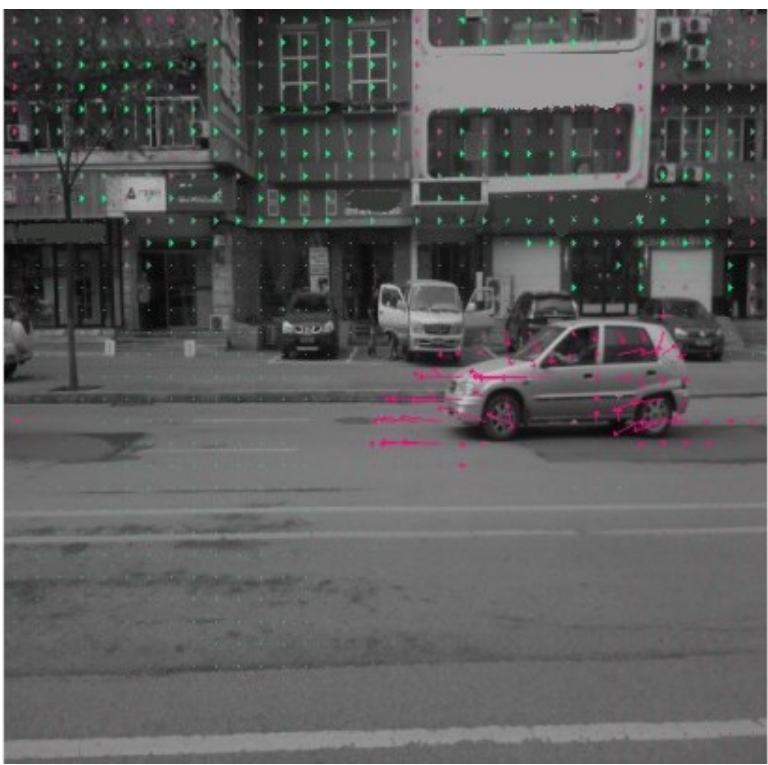

Figure 5. Processed image

Figure 3 and figure 4 are the initial grayscale.Figure 3 is the first frame image,figure 4 is the second frame image,the two images are processed with the program flow chart.It obtain the figure 5 by the processing,from this image it can clear find two kinds of different vectors ,the vectors in the image are not continuous, one kind of them gei together around the car,it can easily find the outline of the moving car,so the purpose of moving target detection is realized.It can also clear see in the image that the direction of the car would run and get the trend of the moving target,so it achieves the purpose of moving target track prediction.

On this platform, it is able to run three frames a second.Because of the calculation of the optical flow 
algorithm is so many that in this processing stage, it cost a lot of time,so the real-time of the system is not good.For improving this situation, Temporal diference method and background subtracion method can be used in som e way.This experiment does not consider a sheltered situation at some moment,if there is Kal man filter algorithm[6],the problem above would be solved,and the prediction of the result would be more quickly.

\section{REFERENCES}

[1] B.D. Lucas and T. Kanade,"An iterative image registration technique with an application to stereo vision",in Proc. Seventh International
Joint Conference on Artificial Intelligence,Vancouver,1981,pp.674679.

[2] M.Proesmans,L.VanGool,E.Pauwels, and

"A.Oosterlinck, Determination of optical flow and its discontinuities using non Computer Vision”, ECCV' 94, 1994, Vo1.2, pp.295-304

[3] Milan S,VaclavH,RogerB. Image processing analysis and machine vision[M]. 2nd edition. Beijing:POSTS\&TELECOM PRESS, 2002.

[4] Gibson J J, Carm ichaelL. The Perception of the Visual World[J]. Science, 1951, 113(2940): 535

[5] J.Y. Bouguet; "Pyramidal implementation of the affine Lucas Kanade featuretracker description of the algorithm",2001.

[6] Huang Chao-qun.Motion detection and tracking based on gaussianmixture model and $\mathrm{k}$ alman filter [D] . Kunming: Yunnan Universi-ty, 2010. 